\title{
A common tumor in an uncommon site: epithelioid Leiomyoma arising from the seminal vesicle-a case report
}

\author{
Xiaoyang Xu, Shuang He, Yangyang Li, Feifei Wen, Lizhen Lư ${ }^{\dagger}$ Zhongze Cui ${ }^{\dagger}$ and Shuhua Wu* (D)
}

\begin{abstract}
Background: Leiomyoma of the seminal vesicle is a rare leiomyoma characterized by the formation of benign leiomyomatous tissue within the seminal vesicle. Although histologically benign, excessive size can lead to urinary system disease if left untreated. Herein, we report a case of a seminal vesicle epithelioid leiomyoma.

Case presentation: A 36-year-old Chinese man sought medical attention at our hospital for urination pain and hemospermia. CT showed a $5.3 \mathrm{~cm} \times 5.0 \mathrm{~cm}$ seminal vesicle mass with a mixed density in the right seminal vesicle. The gross specimen showed light yellow, gray, and white tissues, with softness and hemorrhage in some places. Histologically, it showed classic spindle cell proliferation, with spindle cells arranged in fascicles, and mitosis was rare. Immunohistochemistry showed frequent expression of smooth muscle markers, such as calponin, SMA, and desmin. A diagnosis of epithelioid leiomyoma was proposed according to the immunohistochemical findings and morphology. The patient did not receive adjuvant therapy. There was no evidence of tumor recurrence in the 10 months after surgery.
\end{abstract}

Conclusions: We report the first case of epithelioid leiomyoma in the seminal vesicle. This disease should be included in the differential diagnostic list of seminal vesicle tumors with epithelioid morphology.

Keywords: Seminal vesicle, Epithelioid leiomyoma, Case report, Pathology, Computed tomography

\section{Background}

Primary tumors of the seminal vesicle are rare. Seminal vesicle cysts occur more commonly than solid masses. Among solid masses, the most common primary tumor is adenocarcinoma. Among them, the most common is metastatic adenocarcinoma from colorectal or prostate. Benign solid tumors of the seminal vesicle include cystadenoma of the seminal vesicles, fibroadenoma, solitary fibrous tumor and leiomyoma. Among the seminal vesicle masses, leiomyoma is a very rare mass, with only a few cases reported in the literature. We searched

*Correspondence: wushuhua6108@163.com

'Lizhen Lu and Zhongze Cui contributed equally

Department of Pathology, Binzhou Medical University Hospital,

Binzhou 256603, Shandong Province, China
PubMed using the keywords "leiomyoma", "seminal vesicle", "seminal duct", "seminal vesicle pathology" and "seminal duct pathology". As of January 2021, only 12 cases of seminiferous duct/scrotal leiomyoma have been reported in the medical literature, but no epithelioid leiomyoma has been reported. We report a new case of primary epithelioid leiomyoma of the seminal vesicle and present a brief review of the literature.

\section{Case presentation}

Clinical summary, radiological findings and gross examination

A 36-year-old Chinese man sought medical attention at our hospital for urination pain and hemospermia. The patient developed hematospermia 2 weeks prior. At the same time, there was pain in urination, and the pain 
was a tingling sensation. His symptoms were relieved after urination. There were mixed lesions behind the bladder by B-ultrasonic examination (no image provided). No special disease history was reported.

Computed tomography (CT) showed that the mixed density of mass could be seen in the right seminal vesicle with clear boundary. The size of the mass was $5.3 \mathrm{~cm} \times 5.0 \mathrm{~cm}$. CTU (CT urography) showed that the mass had uneven enhancement, and patchy non enhancement areas could be seen (Fig. 1a-c). Preoperative diagnostic imaging described a "space-occupying right seminal vesicle, potentially malignant disorder".
Next, the patient underwent laparoscopic excision of the seminal vesicle tumor.

Due to the nature of laparoscopic surgery, the specimen was relatively broken. However, it seemed that there was still a fibrous capsule in some places (Fig. 1d). In other word, the boundary of the mass in vivo was relatively clear. The gross specimen showed light yellow, gray, and white tissues, with solid and soft.

\section{Histological characteristics}

Microscopically, the mass was rich in cells, there were fibrous capsules, and the boundary was relatively clear.

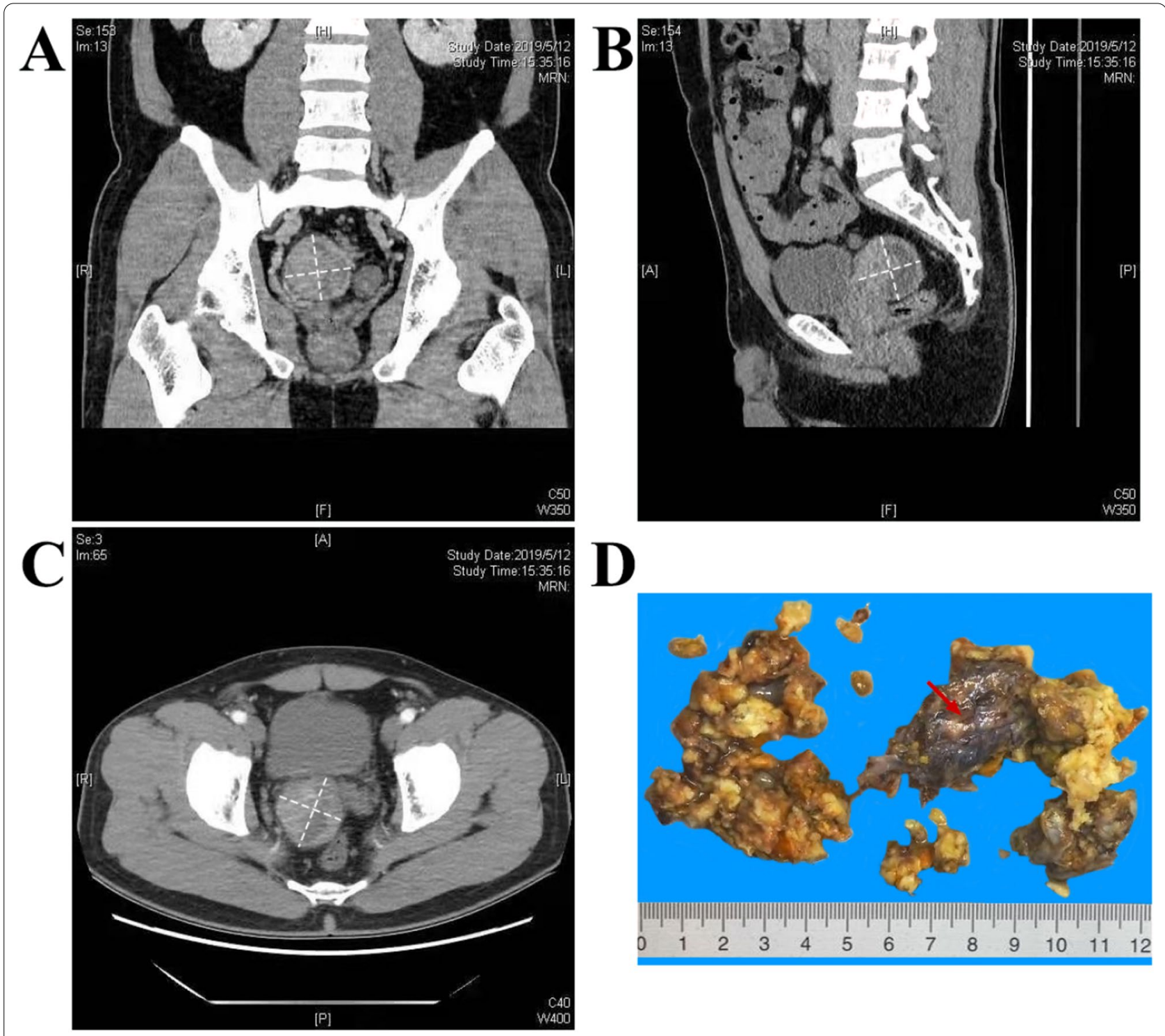

Fig. 1 Clinical imaging findings. a-c CT examination revealed a mixed density mass with a clear boundary. d Macroscopically, the tumor was an $80 \times 55 \times 2-m m$ mass with a fibrous capsule (red arrows) 

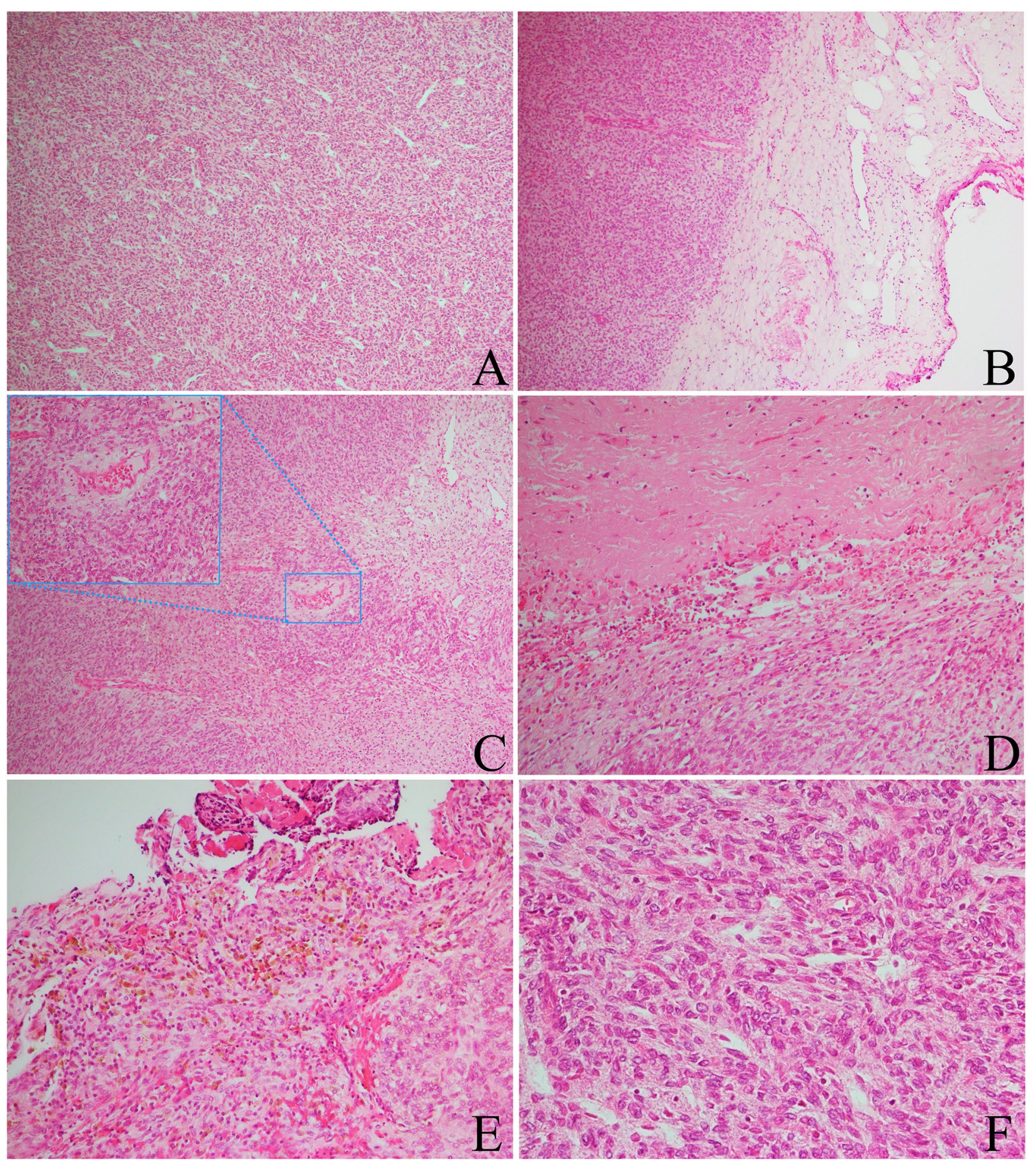

Fig. 2 Histology of the seminal vesicle tumor (H\&E staining, by OLYMPUS BX53). a The tumor cells are dense and arranged like strips ( $\times$ 4). b The tumor has a fibrous capsule and the boundary is relatively clear $(\times 4)$. c Tumor cells line up around blood vessels to form a perivascular tumor-like structure $(x 4)$. $\mathbf{d}$ The collagen with hyaline degeneration could be seen in stroma $(\times 10)$. e Old bleeding can be seen around the tumor $(\times 10)$. $\mathbf{f}$ The tumor is composed of round or polygonal cells and the cytoplasm is eosinophilic or transparent $(\times 20)$ 

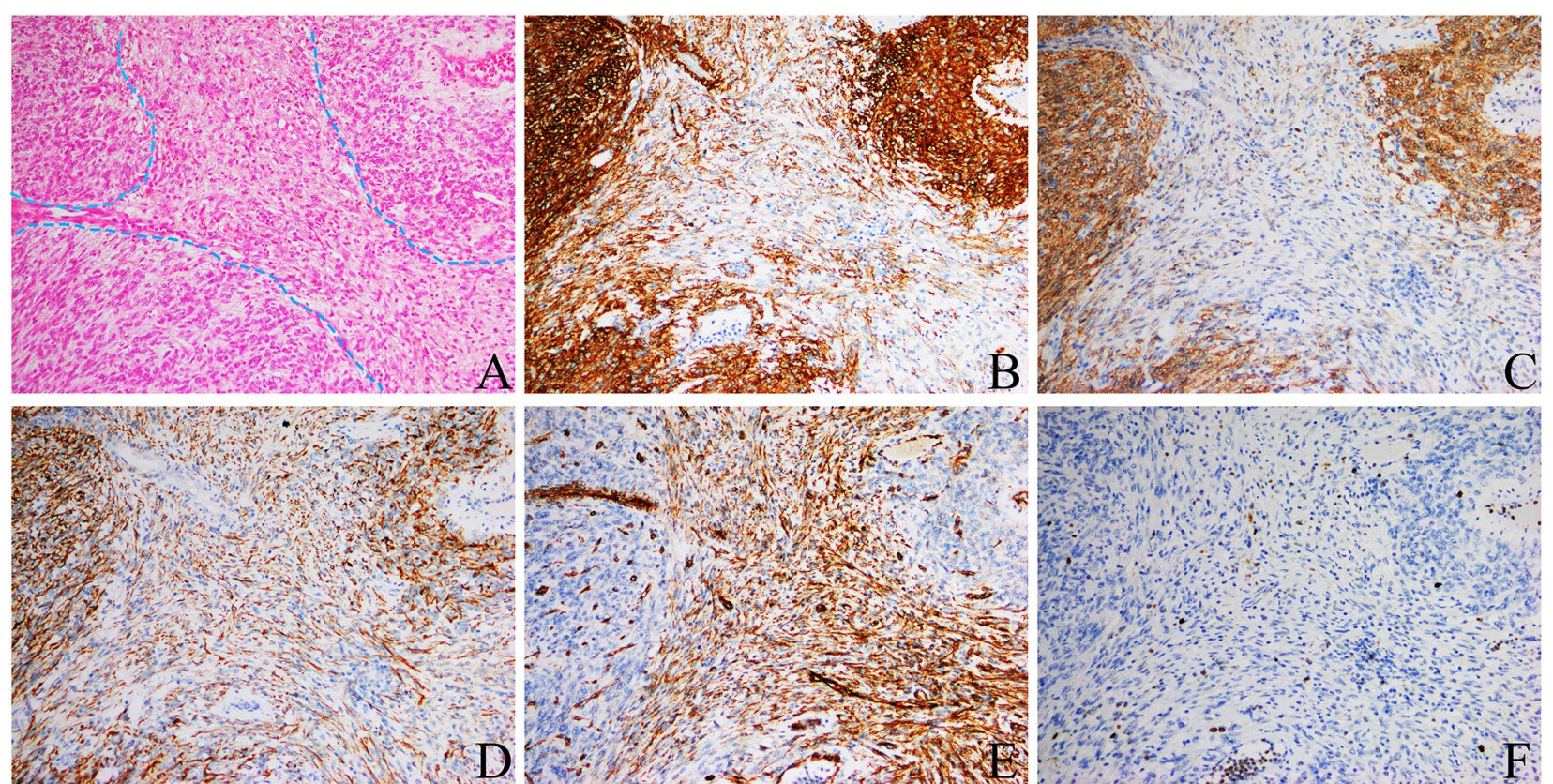

Fig. 3 Immunohistochemistry of seminal vesicle tumor (Envision, $\times 10$, by OLYMPUS BX53). a H\&E staining showed that the tumor cells were denser than interstitial cells. IHC findings showed that most tumor cells were strongly positive for calponin (b) and moderately positive for SMA (c) and desmin (d). None of the tumor cells showed Vimentin expression, while nonneoplastic endothelial cells expressed Vimentin (e). The positive rate of Ki-67 (f) was low

The cells were arranged in the shape of sheets, nests or strips. The tumor cells were round and partly polygonal, with an abundant and acidophilic cytoplasm. The nucleus was located in the center of the cell. Mitosis was rare $(<2 / 10 \mathrm{HPF})$. Some tumor cells lined up around blood vessels to form a perivascular tumor-like structure. Vascular hyperplasia was seen in the stroma (Fig. 2). We found hemosiderin deposits around the tumor, indicating that there was bleeding, which may be the reason for the patient's hemospermia syndrome.

\section{Immunohistochemical findings}

The immunohistochemical findings were CK (locally positive), Vimentin(-), calponin(+), SMA $(+)$, Desmin $(+)$, CK5/6(-), P63(-), HMB45(-), MelanA(-), EMA(-), 34ßE12(-), CD31(-), CD34(-), ERG(-), D2-40(-), S-100(-), CD117(-), DOG-1(-), CD68(-), PSA(-), and Ki-67 (2-5\%) (Fig. 3 and Additional file 1: Fig. S1).

Interpretation of the IHC results. PSA and ERG were negative to rule out metastasis of poorly differentiated prostate cancer. We could exclude low differentiated squamous cell carcinoma because P63 and 34ßE12 were negative. Vascular markers were negative (e.g., CD31, CD34, D2-40, and ERG); therefore, vascular tumors were excluded. HMB45(-), Melan-A(-) and S-100(-) can exclude malignant melanoma. It is not a neurogenic tumor because S-100 was not expressed. GISTs were excluded because CD117, DOG-1 and CD34 were all negative. Ewing's sarcoma was not considered due to ERG negativity. The immunohistochemistry results for smooth muscle markers, such as calponin, SMA, and desmin, were positive. Therefore, the proposed diagnosis was that the patient had a smooth muscle tumor. EMA and CD68 were used to differentiate fibrous histiocytoma from epithelioid leiomyoma.

\section{Discussion and conclusions}

Leiomyoma often occurs in the uterus [1]. It is the most common tumor in the uterus and predominantly originates from smooth muscle. Epithelioid leiomyoma is one of the distinct cell signature subtypes. Epithelioid leiomyoma is mainly composed of round or polygonal cells [2]. The cytoplasm is eosinophilic or transparent. There is a round nucleus and delicate chromatin. Occasionally, the nucleolus can be seen. The cells are arranged in bands, strips and nests. Sometimes tumor cells seem to adhere to each other and occasionally line up around blood vessels to form a perivascular tumor-like structure. Epithelioid cells are the main 


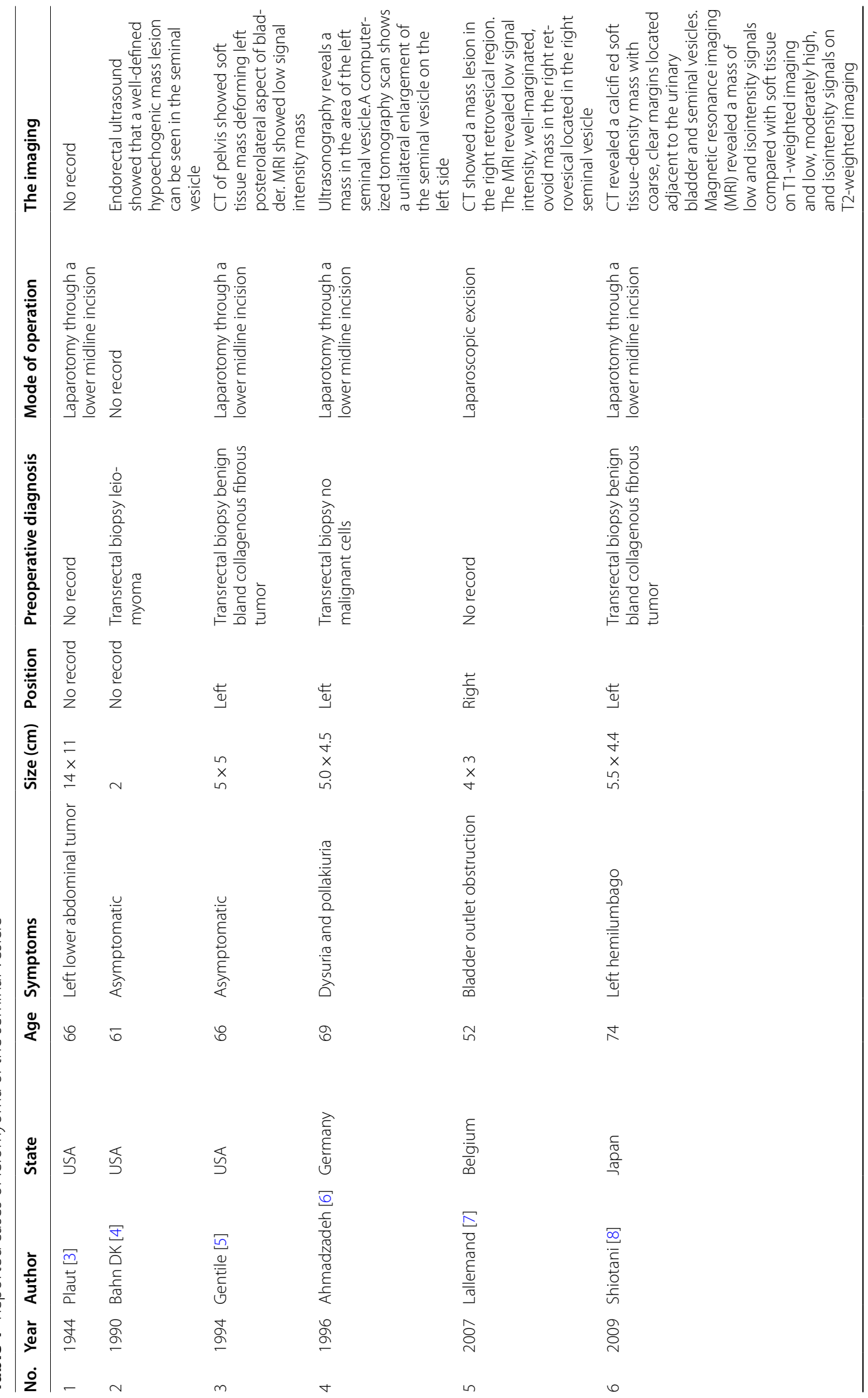




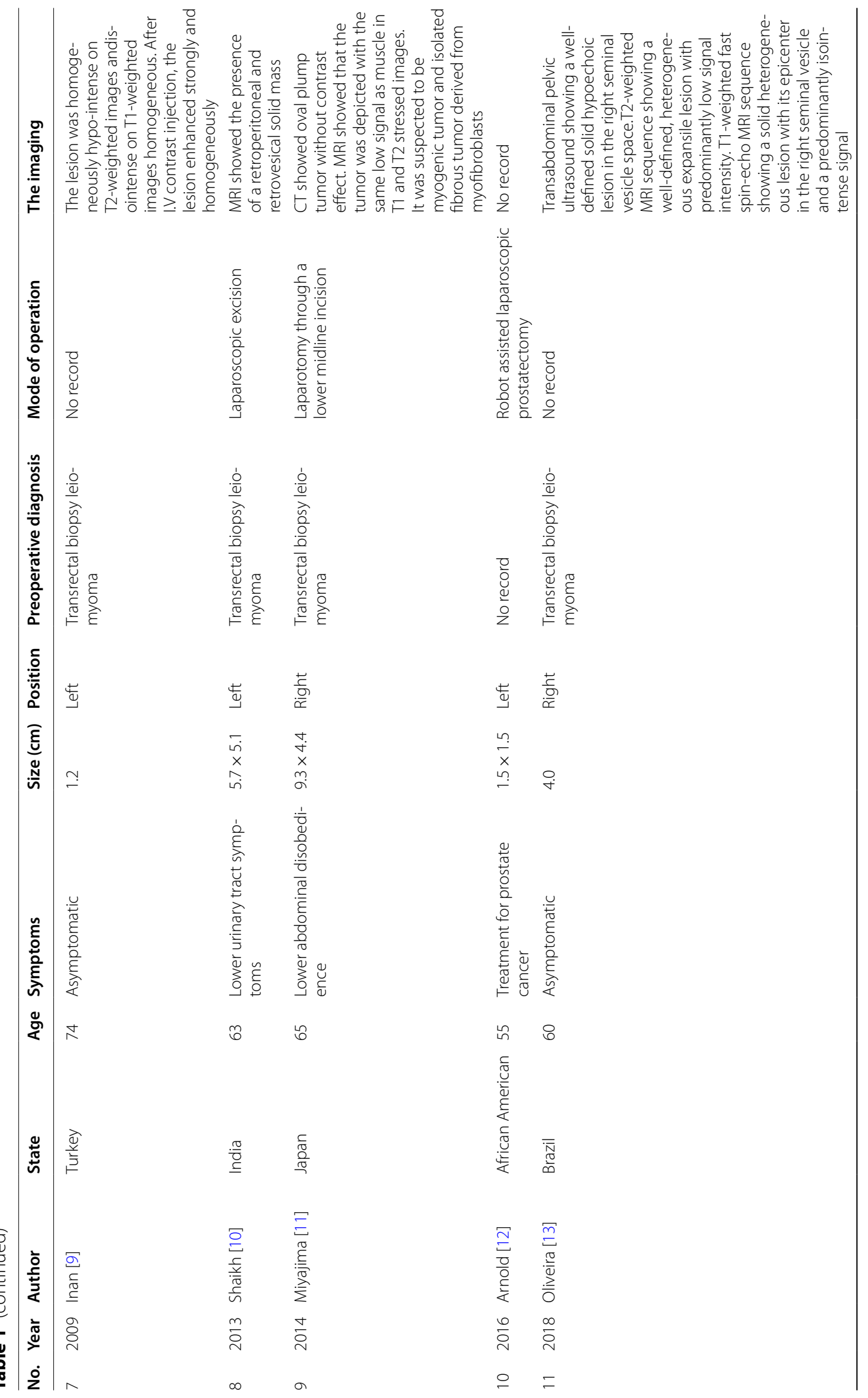




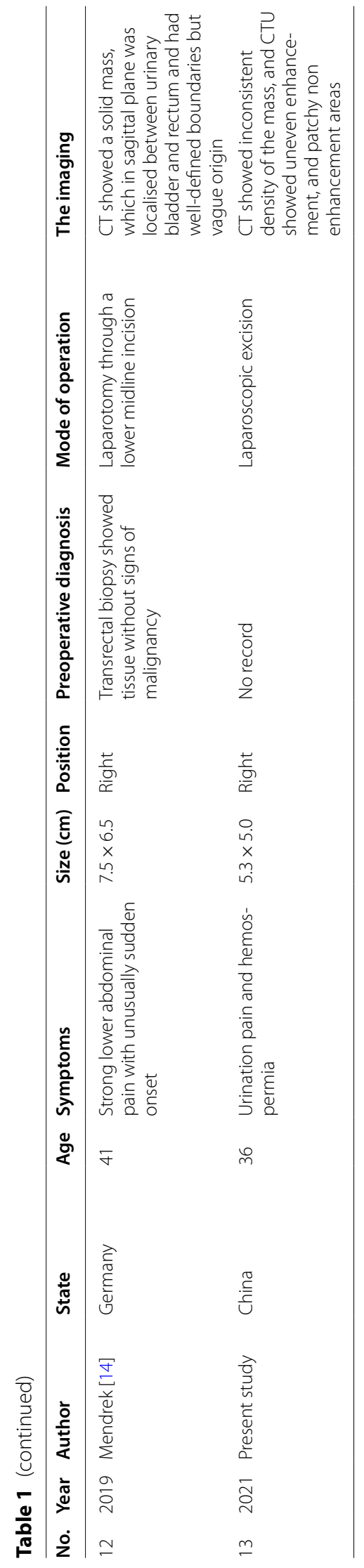


components. Collagen with hyaline degeneration could be seen in the stroma. Studies have found that the cytoplasmic transparency is due to sublethal damage to cells, such as the vacuolation of mitochondria and lysosomes. In some cases, clear cells may be arranged in small nests or tubules, and they could form plexiform tumorlets. Plexiform tumorigenesis is easily misinterpreted as metastatic cancer, particularly metastatic invasive lobular carcinoma (ILC). Most notably, there are cases of death caused by epithelioid leiomyoma in the uterus. Therefore, we believe that epithelioid leiomyoma should be classified as a smooth muscle tumor considering its unique clinicopathological characteristics and malignant potential.

Diseases of the seminal vesicle are extremely rare, and there are only a few reports in the literature. A systematic review of the medical literature shows that only 12 cases of leiomyoma of the seminal vesicle have been reported so far (Table 1). All the reports agree that it is a benign tumor, and there were no recurrences or metastases reported. The median age of the patients was 65 years, and the youngest age of onset was 36 years. When the tumor is small, there are no spontaneous symptoms. However, when the tumor is more than $10 \mathrm{~cm}$, it can cause lower abdominal pain and urinary symptoms. For radiologic examination, CT or MRI investigation is necessary. In 12 cases found in literature review, 10 cases underwent a radiological clarification either with CT or with MRI had been performed before any further intervention. In 2 cases based on MRI, the definitive diagnosis such as a solitary fi brous tumor or leiomyoma could be suspected, but at the end in all these cases, the diagnosis was only confirmed after the extirpation. At present, there are no publications on systematic radiology standards that can be used for the examination of such cases.

This is the first report of epithelioid leiomyoma in the seminal vesicle, and the origin of the mass was identified by several groups of immunohistochemical markers. The most common treatment for seminal vesicle leiomyoma is surgery, which can not only relieve symptoms but also provide a clear diagnosis. In previous reports, open surgery was most frequently performed, and a few patients underwent laparoscopic resection. To date, there have been no reports of recurrence of leiomyoma in the seminal vesicle. The author will continue to follow up this case.

\section{Abbreviations}

CK: Cytokeratin; CT: Computed tomography; SMA: Smooth muscle actin; EMA: Epithelial membrane antigen.

\section{Supplementary Information}

The online version contains supplementary material available at https://doi. org/10.1186/s12894-022-00963-3.

Additional file 1: Fig. S4 The immunohistochemical findings were SMA(+), calponin(+), Ki-67 (2-5\%), CK (locally positive), PSA(-), ERG(-), CK5/6(-), P63(-), 34ßE12(-), HMB45(-), MelanA(-), S-100(-), CD68(-), $\operatorname{EMA}(-), \operatorname{CD} 117(-), \mathrm{DOG}-1(-), \mathrm{CD} 34(-), \mathrm{D} 2-40(-)$ and CD31(-).

\section{Acknowledgements \\ None.}

\section{Authors' contributions}

$X Y$ designed and drafted the manuscript. HS, LY and WF analysed and interpreted of data. LZ and CZ collected clinical data. WHS substantively revised it and approved the final manuscript.

\section{Funding}

National Natural Science Foundation of China (No.81772637); Scientific Research Staring Foundation of Binzhou Medical University (NO.BY2019KJ16). The funders are responsible for the publication expenses of this study and don't participate in any experiments and conclusions related to this study.

Availability of data and materials

All data generated or analyzed during this study are included in this published article and its supplementary information files.

\section{Declarations}

\section{Ethics approval and consent to participate}

This case study was approved by the Institutional Review Board for ethical committee of Binzhou Medical University Hospital.

\section{Consent for publication}

The patient gave has written consent for image and data publication.

\section{Competing interests}

The authors declare that they have no competing interests.

Received: 27 August 2021 Accepted: 24 January 2022

Published online: 29 January 2022

\section{References}

1. Stewart EA, Laughlin-Tommaso SK, Catherino WH, et al. Uterine fibroids. Nat Rev Dis Primers. 2016;23:16043.

2. Karpathiou G, Papoudou-Bai A, Clemenson A, et al. The uterine plexiform lesions revisited. Am J Clin Pathol. 2020;154(2):178-89.

3. Plaut A, Standard S. Cystomyoma of seminal vesicle. Ann Surg. 1944;119(2):253-61.

4. Bahn DK, Brown RK, Shei KY, et al. Sonographic findings of leiomyoma in the seminal vesicle. J Clin Ultrasound. 1990;18(6):517-9.

5. Gentile AT, Moseley HS, Quinn SF, et al. Leiomyoma of the seminal vesicle. J Urol. 1994;151(4):1027-9.

6. Ahmadzadeh $M$, Bosse A. Leiomyoma of the seminal vesicle. Urol Int. 1996;57(4):252-4.

7. Lallemand B, Busard P, Leduc F, et al. Laparoscopic resection of a leiomyoma of the seminal vesicle. Indian J Urol. 2007;23(1):70-1.

8. Shiotani T, Kawai N, Sato M, et al. Leiomyoma of the seminal vesicle. Jpn J Radiol. 2009:27(5):218-20.

9. Inan N, Fayda M, Aksu G, et al. Leiomyoma of the seminal vesicle mimicking tumoral extension of prostatic carcinoma. Acta Chir Belg. 2009;109(6):811-4.

10. Shaikh AS, Bakhshi GD, Khan AS, et al. Leiomyoma of the seminal vesicle: a rare case. Clin Pract. 2013;3(2):e32.

11. Miyajima S, Irie S, Nakamura N, et al. A case of leiomyoma of the seminal vesicle. Nihon Hinyokika Gakkai Zasshi. 2014;105(3):144-8 Japanese. 
12. Arnold SJ, Lin FC, Eldersveld JM, et al. Seminal vesicle leiomyoma mimicking extra-prostatic extension of prostatic adenocarcinoma. Urol Case Rep. 2016;6:18-20

13. Oliveira TS, Stamoulis DNJ, de Souza LRMF, et al. Leiomyoma of the seminal vesicle. Radiol Bras. 2018;51(3):200-1.

14. Mendrek M, Bach C, Gaisa NT, et al. Leiomyoma arising from the right seminal duct/seminal vesicle-report of a rare case and review of the literature. Andrologia. 2019;51(2):e13174.

\section{Publisher's Note}

Springer Nature remains neutral with regard to jurisdictional claims in published maps and institutional affiliations.

- fast, convenient online submission

- thorough peer review by experienced researchers in your field

- rapid publication on acceptance

- support for research data, including large and complex data types

- gold Open Access which fosters wider collaboration and increased citations

- maximum visibility for your research: over 100M website views per year

At BMC, research is always in progress.

Learn more biomedcentral.com/submissions 